\title{
Acquisition and retention of visual discrimination learning after ablation of perirhinal cortex in the rat
}

\author{
M. J. EACOTT \\ University of Durham, Durham, England
}

\begin{abstract}
Eight Dark Agouti rats were trained in a version of concurrent discrimination learning in a computercontrolled automated apparatus that allowed the use of complex abstract patterns as stimuli. Preoperatively, they learned a five-pair concurrent discrimination and were tested for their postoperative retention of this discrimination. Rats with ablation of the perirhinal cortex were impaired in their retention of the concurrent discriminations. However, they were totally unimpaired in postoperative acquisition of two new concurrent discrimination sets. Moreover, they were also completely unimpaired in postoperative retention of these postoperatively learned discrimination sets. The deficit therefore appears to be a specific retrograde amnesia, with no evidence for anterograde effects.
\end{abstract}

The importance of the perirhinal cortex in performance of matching- and non-matching-to-sample in the monkey has become increasingly clear over the last 10 years. Monkeys with perirhinal or combined perirhinal and entorhinal cortex lesions fail to acquire or to perform delayed matchto-sample efficiently (Eacott, D. Gaffan, \& Murray, 1994; D. Gaffan \& Murray, 1992; Horel, Voytko, \& Salsbury, 1984; Meunier, Bachevalier, Mishkin, \& Murray, 1993; Zola-Morgan, Squire, Clower, \& Rempel, 1993). Ablation of the perirhinal cortex alone is sufficient to cause this deficit. However, the initial interpretation of this failure as an impairment of visual recognition memory has become increasingly less clear, as it has become apparent that these animals may have deficits in tasks other than those that rely on recognition memory. For example, following ablation of the perirhinal cortex, monkeys may also be poor at concurrent discrimination learning (D. Gaffan, 1994; Horel, Pytko-Joiner, Voytko, \& Salsbury, 1987; Buckley \& D. Gaffan, 1997), a task that is not generally considered to be reliant on visual recognition memory. Deficits can also be seen in the learning of visual-visual associations following lesions to the perirhinal cortex (Murray, D. Gaffan, \& Mishkin, 1993), again a task that is not thought to rely heavily on recognition memory. Moreover, following ablation of the perirhinal and entorhinal cortex, monkeys may also be completely unimpaired on tasks that do stress visual recognition memory, for example, a difficult test of recency memory (Eacott et al., 1994), which is tested in a manner identical to the test of recognition memory on which they are so impaired. Thus, the simple view of the perirhinal

This work was supported by a grant from the Medical Research Council. I gratefully acknowledge the help of Angela Spark, who tested the rats, and Shirley Whiteley, who processed the histology. Correspondence concerning this article should be addressed to M. J. Eacott, Department of Psychology, Science Laboratories, University of Durham, South Road, Durham DH1 3LE, England (e-mail: mjeacott@durham.ac.uk). cortex as a visual recognition processor is increasingly difficult to maintain.

A similar picture emerges from the study of the perirhinal cortex in the rat. The cytoarchitectonic and anatomical similarities between the cortex surrounding the rhinal sulcus in the rat and the monkey are strong (Burwell, Witter, \& Amarel, 1995), although some minor differences have also been noted (Burwell et al., 1995). Moreover, the electrophysiological responses of the neurons within the area of the two species is also similar (Zhu \& Brown, 1995; Zhu, Brown, \& Aggleton, 1995). Most importantly, the cortex in and around the rhinal sulcus also plays a part in the efficient performance of recognition memory tasks in rats. Ablation of this tissue results in a deficit in a matchingto-sample task using either visual stimuli (Mumby \& Pinel, 1994) or odors (Otto \& Eichenbaum, 1992). However, as in the monkey, increasingly there are reports that a simplistic interpretation of these findings in the rat should be guarded against. For example, as in the monkey, deficits in associative learning following lesions of the perirhinal cortex are observed in the rat (Bunsey \& Eichenbaum, 1993). Moreover, the deficits that are reported to follow lesions to this cortex are not always consistent. For example, there are reports of both impaired discrimination learning (Astur, Mumby, \& Sutherland, 1995; Wiig, Booth, Lui, Cooper, \& Bilkey, 1995) and unimpaired discrimination learning (Kornecook, Lui, Duva, Anzarat, \& Pinel, 1995) following damage to the perirhinal cortex. There are doubtless many differences between the procedures and the stimuli used in these tasks, but from the data available it is not clear that the results covary with any of these parameters. Astur et al.'s (1995) study, for example, included preoperative training, while the rats in Wiig et al.'s, (1995) and Kornecook et al.'s (1995) studies were not trained preoperatively. Kornecook et al. included a concurrent discrimination in addition to the simple twochoice discriminations used by Astur et al. and Wiig et al. 
The importance of concurrent over simple two-choice discrimination learning is emphasized by Buckley and D. Gaffan's (1997) findings that deficits after lesions to the perirhinal cortex in the monkey may be seen when the discrimination is made more complex by increasing the number of foils among which a choice has to be made. However, in the rat, Kornecook et al. found no deficit with this more difficult concurrent learning, although other laboratories found deficits using the simpler two-choice methodology.

The above discussion emphasizes that despite the robust impairment in matching-to-sample which follows damage to the perirhinal cortex in rats and monkeys, we do not yet fully understand the basis of the impairment. Deficits in match-to-sample could plausibly be seen for a variety of reasons, including perceptual, mnemonic, and motivational impairments. Therefore, there is a need to more fully identify the deficit that follows perirhinal ablation in the rat. The current investigation examines the role of the perirhinal cortex in concurrent discrimination learning in a computer-controlled testing apparatus. This provides the opportunity to present the animals with a series of discriminations in a well-controlled testing environment. This testing apparatus allows us much greater confidence that the animals are relying on visual cues associated with each stimulus rather than on an amalgam of visual, odor, and somatosensory cues normally available to the rat in a traditional testing apparatus. This brings the testing situation much closer to that used in automatic testing in the monkey (e.g., D. Gaffan, 1994; Murray et al., 1993) and allows us to specify that visual memory processes are the object of the study. In this study, the retention of both preoperatively and postoperatively learned concurrent discriminations and the acquisition of postoperatively learned concurrent discriminations are examined.

\section{METHOD}

\section{Subjects}

Ten Dark Agouti rats (Bantin and Kingman, Hull, U.K.) were used in this experiment. All were housed individually under diurnal conditions (12:12-h light:dark) and were tested during the light period of the cycle. The animals were tested 5 days a week, and on the day prior to testing were fed a restricted amount of laboratory chow. Except when in the testing apparatus, the rats had free access to water. All had previously attempted to learn a series of difficult visual discriminations in the apparatus described without marked success. This task was then abandoned, and the present experiment was substituted. At the start of the current experiment, the rats weighed approximately $250 \mathrm{~g}$. One rat failed to survive surgery and another died of respiratory infection in the days following surgery. Thus, a total of 8 rats completed the experimental testing; 4 of them had bilateral perirhinal lesions and 4 had bilateral sham surgery.

\section{Apparatus}

The rats were tested in a computer-controlled apparatus which has been described in detail elsewhere (E. A. Gaffan \& Eacott, 1995). Briefly, it consists of a Y-shaped area, the ends of the arms of which are formed by two display monitors in the shape of a shallow V. Complex patterns can be displayed on the monitors to served as visual stimuli. Rats make choices between stimuli displayed in two arms by entering one arm and approaching the stimulus displays.
Approach is detected by photodetector beams that cross the arms of the maze. Approach of the correct display causes a reward pellet to be automatically dispensed into a hopper situated between the two display monitors. A single reward is dispensed immediately, followed by a second pellet approximately $2 \mathrm{sec}$ later. When the rat pushes open the door of the hopper to retrieve the second pellet, the correct stimulus disappears. Approaching the incorrect display causes both stimuli to disappear, leaving a blank screen for the intertrial interval of approximately $4 \mathrm{sec}$. The next trial begins approximately $2 \mathrm{sec}$ later, as long as the rat remains in the arm. Should the rat leave this arm following either a correct or incorrect response, the next trial commences only when he returns to that arm.

\section{Procedures}

Behavioral training. The rats were trained in this maze prior to surgery. The initial training to respond to stimuli presented on the monitors and to retrieve pellets is described elsewhere (E. A. Gaffan \& Eacott, 1995). Following this basic training, the rats were trained, without success, in a difficult discrimination task between stimuli similar to those used in the currently reported experiment. In that task, the animals performed a series of two-choice discriminations each for a single session consisting of a maximum of 100 trials. Since they failed to progress satisfactorily, the task was abandoned and the rats entered the current experiment, a concurrent discriminationlearning task.

Preoperative testing. There were two sets of stimuli, Set 1 and Set 2. The stimuli in each set were chosen at random from a set of 525 stimuli generated using a stimulus-generating algorithm described elsewhere (E. A. Gaffan \& Eacott, 1995). The sets were then checked to ensure that a stimulus did not appear in both sets. Each set consisted of five pairs of complex abstract stimuli. Within each pair, 1 stimulus was arbitrarily designated as correct $(\mathrm{S}+)$ and the other as incorrect $(\mathrm{S}-$ ). Initially the task used only one pair. On each trial, the $\mathrm{S}+$ was displayed in one arm and the $\mathrm{S}-$ in the other and the rats made a choice between them. Rewards for correct choices were delivered as described above. There was a maximum of 100 trials in each session. When the animals reached a criterion of $80 \%$ within a single session, criterion had been reached and the second pair was introduced. Each subsequent trial consisted of one $S+$ and one $S-$, although each could come from either pair, with the sole restriction that equal numbers of trials with each $\mathrm{S}+$ were presented within the full 100-trial session. The animals continued the discrimination learning with two pairs until a criterion of $80 \%$ correct in trials with the S+ from Pair 1 and $80 \%$ of trials with the S+ from Pair 2 was achieved. At this point, the third pair was introduced in exactly the same way as before until the criterion of $80 \%$ in trials with each of the three $S+s$ was reached. Subsequent pairs were introduced as before until all five pairs were in use. At this point, a session consisted of a total of 100 trials, 20 trials with each of the $S+s$, which could in turn be presented with any of the five $S-s$. When the animal achieved $80 \%$ with all $S+s$ with five pairs, criterion had been reached. The animals then underwent surgery, either bilateral perirhinal ablation or sham surgery.

Surgery. Selective bilateral aspiration lesions of the perirhinal cortex were made under visual guidance using an operating microscope. The intended extent of the lesion was determined according to the Paxinos and Watson (1986) atlas. The rats were maintained under halothane anesthesia throughout the procedure. The head was shaved and the rat was placed in a stereotaxic head holder that allowed for rotation of the head. The scalp was cut and the temporal muscle retracted to reveal the skull that overlies the rhinal fissure. Rotating the head holder to allow access, a dental drill was used to remove a portion of the skull approximately 4-7 $\mathrm{mm}$ posterior to bregma. The underlying dura was slit to allow aspiration of the perirhinal cortex. The overlying muscle was returned to position, the wound was closed using wound clips, and powder containing $20 \%$ chloramine was applied. Each rat was subcutaneously injected with $5 \mathrm{ml}$ of saline, $0.3 \mathrm{ml}$ of millophyline, and $0.05 \mathrm{ml}$ of Temgesic. The 


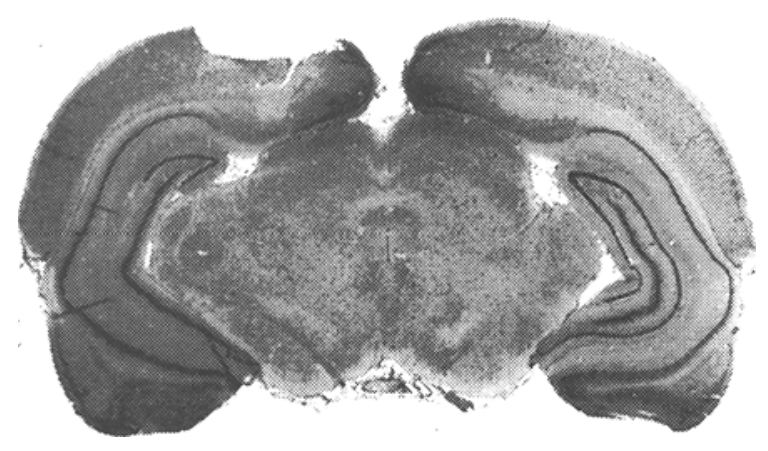

Figure 1. A representative section showing a bilateral perirhinal lesion from Animal R35.

sham surgery was exactly as above with the exception that no aspiration of cortex took place. The dura was slit and the wound then closed as above. Recovery from surgery was uneventful in all cases reported here, although 1 animal failed to survive surgery and another animal died of respiratory infection in the days following surgery.

At the end of the study, the rats were perfused intracardially with $5 \%$ formol saline. The brains were subsequently blocked, embedded in wax, and cut in 10- $\mu$ coronal sections. Every 10 th section was stained with a Nissl stain (cresyl violet).

Postoperative retention of preoperative learning. Following surgery and a recovery period of 2 weeks, the animals were tested for their retention of the preoperatively learned five-pair concurrent discrimination. All five pairs were simultaneously introduced, and testing continued until criterion was reattained.

Postoperative acquisition of concurrent discrimination learning. Following retention testing, all animals were introduced to a second five-pair concurrent leaning set. The animals that had previously learned Set 1 now learned Set 2 , and vice versa. The discrimination was introduced one pair at a time, as in preoperative acquisition, until criterion as above was reached. When they had learned this concurrent discrimination set, all rats learned a third set of five pairs of concurrent discrimination learning to criterion (Set 3 ) exactly as before.

Postoperative retention of postoperative learning. Finally, animals were tested for postoperative retention of the postoperatively learned discriminations. First they were tested after a 2-week delay on retention of the last learned set, Set 3 . Finally, they were tested on the other postoperatively learned discrimination, either Set 1 or Set 2, depending on the animal.

\section{RESULTS}

\section{Histological Results}

In all cases, the lesions were essentially as intended. Figure 1 illustrates a representative section showing a bilateral perirhinal lesion from Animal R35. Figure 2 shows the extent of a relatively small (R28) and a relatively large lesion (R34). As can be seen, there was very little damage outside the intended region of the lesion. In particular, there was very little encroachment ventrally into entorhinal cortex, although there was some minor unintended damage on the dorsal edge of the intended lesion, damaging the temporal visual cortex. The perirhinal cortex was severely damaged in all cases, although note that there was also some degree of intact perirhinal tissue, particularly at the dorsal extent of the intended lesion.

\section{Behavioral Results}

Preoperative acquisition. As can be seen from Table 1, there was no difference between the groups preoperatively, either in their trials to criterion $(U=5, p=.486)$ or the number of errors to criterion $(U=6, p=.686$ ). (In these and subsequent statistical analyses, a Mann-Whitney $U$ test was used as an appropriate nonparametric test.) In fact, the group that subsequently became the perirhinal group actually performed slightly, although not significantly, better overall. However, it is clear that Set 1 is substantially harder than Set 2 to acquire (trials, $U=1, p=$ .058 ; errors, $U=0, p=.028$ ), regardless of group. These rats had previously failed to learn a different discrimination task in the same apparatus, and comparison with the performance of naive rats indicates that this experience substantially slowed their learning of these discriminations (Eacott, unpublished observations).

Postoperative retention of preoperatively learned set. The postoperative retention of the discrimination is shown in Figure 3. From this figure, it is apparent that the perirhinal group madc more errors and required more trials to reacquire this discrimination postoperatively than did the sham-operated group. This difference between the groups is significant, both in trials to criterion $(U=0, p=$ $.014)$ and in errors to criterion $(U=1, p=.029)$. (In these and all subsequent postoperative comparisons between the groups, one-tailed probabilities are given, since the clear prediction under test is that the perirhinal group will be impaired.) The difference between Sets 1 and 2, however, is not evident in the retention data, since within both groups



Figure 2. The extent of a relatively small (R28, dotted) and a relatively large (R34, vertical stripes) lesion drawn onto standard brain sections taken from Paxinos and Watson (1986). 
Table 1

Pre- and Postoperative Acquisition of Concurrent Discrimination Sets Following Peripheral Ablation

\begin{tabular}{|c|c|c|c|c|c|c|c|c|c|}
\hline \multirow[b]{3}{*}{ Rat } & \multirow[b]{3}{*}{ Set } & \multirow{2}{*}{\multicolumn{2}{|c|}{$\begin{array}{l}\text { Preoperative } \\
\text { Acquisition }\end{array}$}} & \multicolumn{6}{|c|}{ Postoperative Acquisition } \\
\hline & & & & \multicolumn{2}{|c|}{ Acq. 2} & \multicolumn{2}{|c|}{ Acq. 3} & \multicolumn{2}{|c|}{ Total $(2+3)$} \\
\hline & & Trials & Errors & Trials & Errors & Trials & Errors & Trials & Errors \\
\hline \multicolumn{10}{|c|}{ PRh } \\
\hline $\mathrm{R} 26$ & 1 & 2,026 & 502 & 726 & 134 & 1,555 & 392 & 2,281 & 526 \\
\hline $\mathrm{R} 28$ & 2 & 1,046 & 270 & 1,109 & 246 & 991 & 249 & 2,100 & 495 \\
\hline $\mathrm{R} 34$ & 1 & 1,514 & 439 & 426 & 79 & 1,740 & 384 & 2,166 & 463 \\
\hline $\mathrm{R} 35$ & 2 & 1,325 & 320 & 1,399 & 414 & 2,212 & 519 & 3,611 & 933 \\
\hline Mean & & $1,477.75$ & 382.75 & 915 & 218.25 & $1,624.5$ & 386 & $2,539.5$ & 604.25 \\
\hline \multicolumn{10}{|c|}{ Sham } \\
\hline R29 & 2 & 1,535 & 359 & 1,169 & 285 & 1,976 & 507 & 3,145 & 792 \\
\hline R30 & 1 & 2,376 & 620 & 831 & 160 & 577 & 411 & 1,408 & 571 \\
\hline R31 & 1 & 2,193 & 596 & 1,160 & 210 & 1,723 & 468 & 2,883 & 678 \\
\hline $\mathrm{R} 32$ & 2 & 815 & 173 & 1,224 & 296 & 900 & 256 & 2,124 & 552 \\
\hline Mean & & $1,729.75$ & 437 & 1,096 & 237.75 & 1,294 & 410.5 & 2,390 & 648.25 \\
\hline
\end{tabular}

Note-The first-leamed set was either Set 1 or Set 2, as indicated under the column headed Set. Shown under Acq. 2 are the data for acquisition of the other set. Finally, all animals learned Set 3.

the animals reacquiring the more difficult Set 1 overlap in performance with those reacquiring the easier Set 2.

Postoperative acquisition of new sets. As Table 1 shows, however, the difference between the groups was not maintained in postoperative acquisition of a new set of five pairs (trials, $U=5, p=.243$; errors, $U=6, p=.343$ ), in which the perirhinal group seemed slightly, though insignificantly, better than the sham-operated group, as had been the case before surgery. Postoperative acquisition of a second five-pair concurrent discrimination confirmed this finding, there being no difference between the two groups in either measure (trials, $U=5, p=.243$; errors, $U=6, p=.343$ ). Even when the results from the two postoperative sets are combined in order to average out possible idiosyncratic effects of particular sets, there are no differences between the groups (trials, $U=7, p=.443$; errors, $U=4, p=.171$ ).

Postoperative retention of postoperative learned discrimination. Postoperative retention of postoperative learned discriminations was also tested and is shown in Table 2. There was again no difference between the groups in their retention of Set 3 (trials, $U=8, p=.557$; errors, $U=7.5, p=.5$ ) or the other postoperatively learned set (trials, $U=5.5, p=.293$; errors, $U=7, p=.443$ ). Again, combining the results of the retention of the two sets to diminish the effects of idiosyncrasies revealed no suggestion of an impairment (trials, $U=8, p=.557$; errors, $U=8$, $p=.557)$.

\section{DISCUSSION}

The animals learned a five-pair concurrent discrimination preoperatively and were tested for their postoperative retention of this discrimination. There was a significant impairment in the perirhinal group. However, this group was not impaired in subsequent acquisition of two new sets of similar discriminations or in retention of postoperatively acquired discriminations. Thus, the impairment appears to be a specific retrograde amnesia for preopera- tively learned discrimination sets, with no associated anterograde effects.

A clear deficit in retention of preoperative learning was found. This was despite the fact that preoperatively the rats were learning one of two different concurrent discrimination sets and, by chance, one set proved markedly more difficult to learn than the other. Postoperatively, this difference was less marked but still apparent, and the only overlap between the groups was the worst sham-operated rat tested on the more difficult set and the least impaired operated rat tested on retention of the easier discrimination set. Over all data where the effect of set difficulty is balanced between groups, the mean of the operated group is over twice the mean of the sham-operated group. Thus, the difference between the groups is marked and the full extent of the deficit in postoperative retention of a preoperatively learned discrimination is, in part, hidden by the effect of set difficulty.



Figure 3. The mean postoperative number of trials (left) and errors (right) to criterion in retention of the preoperatively learned discrimination set. The dots show the data for the individual rats. The numbers beside the individual data points indicate the number of trials or errors that were beyond the scale. 
Table 2

Postoperative Retention of Pre- and Postoperatively Learned Concurrent Discrimination Sets Following Perirhinal Ablation

\begin{tabular}{|c|c|c|c|c|c|c|c|c|}
\hline \multirow[b]{3}{*}{ Rat } & \multirow{2}{*}{\multicolumn{2}{|c|}{$\begin{array}{c}\text { Retention of } \\
\text { Preop. Learning } \\
\end{array}$}} & \multicolumn{6}{|c|}{ Retention of Postoperative Learning } \\
\hline & & & \multicolumn{2}{|c|}{ Set 3} & \multicolumn{2}{|c|}{ Other Postop. Set } & \multicolumn{2}{|c|}{ Total } \\
\hline & Trials & Errors & Trials & Errors & Trials & Errors & Trials & Errors \\
\hline \multicolumn{9}{|c|}{ PRh } \\
\hline R26 & 311 & 66 & 260 & 55 & 281 & 71 & 441 & 126 \\
\hline R28 & 866 & 127 & 231 & 45 & 250 & 56 & 481 & 101 \\
\hline $\mathrm{R} 34$ & 330 & 63 & 205 & 43 & 95 & 25 & 300 & 68 \\
\hline R35 & 279 & 44 & 337 & 68 & 637 & 154 & 974 & 222 \\
\hline Mean & 446.5 & 75 & 258.25 & 52.75 & 315.75 & 76.5 & 549 & 129.25 \\
\hline \multicolumn{9}{|c|}{ Sham } \\
\hline R29 & 152 & 32 & 348 & 69 & 124 & 35 & 472 & 104 \\
\hline R30 & 132 & 23 & 156 & 27 & 237 & 60 & 393 & 87 \\
\hline R31 & 230 & 54 & 966 & 199 & 281 & 76 & 1,247 & 275 \\
\hline R32 & 164 & 23 & 171 & 43 & 231 & 53 & 402 & 96 \\
\hline Mean & 169.5 & 33 & 410.25 & 84.5 & 218.25 & 56 & 628.5 & 140.5 \\
\hline
\end{tabular}

Note-All rats were tested for retention of Set 3 and for either Set 1 or Set 2, depending on which was learned postoperatively. See Table 1 for further details of set.

The lesion causing this impairment was intended as a perirhinal lesion, and the histological analysis revealed that the perirhinal cortex was indeed severely damaged in all cases. However, there was some intact tissue left in some cases, and so it is possible that an even more severe deficit may be revealed by more complete perirhinal lesions. However, performance did not obviously vary with the extent of the lesions in the presently reported study. Histological analysis also revealed that there was some minor encroachment into the area dorsal to the intended lesion area, area TE3. Area TE3 is a temporal visual area, and lesions to this area might be expected to have a detrimental effect on performance of the visual discrimination tasks used here. However, it is unlikely that the minor damage seen to TE3 is the cause of the deficits reported here since the damage was relatively minor. Thus, the impairment seen can be reliably ascribed to the damage to the perirhinal cortex.

This result adds to that already reported for the rat following lesions of the perirhinal cortex. There have previously been mixed reports about the presence or absence of a deficit following similar lesions. However, all previous reports have involved hand testing with discriminanda that could be identified by visual, tactile, and olfactory means. The automated, computer-controlled apparatus used in the present experiment avoids such possible confounds. The deficit reported here is therefore reliably in the discrimination of visually presented stimuli. One other difference between previous reports and the present experiment is that a relatively taxing version of current discrimination learning was used in the present study. In this version, any $\mathrm{S}+$ could appear with any $\mathrm{S}-$. The result of this is that an $\mathrm{S}+$ has to be distinguished not only from a single $\mathrm{S}-$, but from any of the five $S-s$ with which it could be paired. This means that the stimuli must be identified more accurately in the present version than in the simple situation in which it must be readily distinguished from only a single
S-. The importance of this factor may be seen in Buckley and D. Gaffan's (1997) study, which showed a similar impairment in retention of preoperatively learned discriminations in the monkey following perirhinal lesions. Initially, they showed no impairment in postoperative acquisition of a new set of discriminations. However, the deficit was reinstated when the postoperative testing was made more difficult by increasing the difficulty of the discrimination and the number of discriminations to be learned. Thus, the relative difficulty of our concurrent learning task could be the important factor that allowed a deficit to be seen in this task.

However, in complete contrast to this effect on retention of preoperatively learned discrimination sets, there is no hint of a difference between the groups in their postoperative acquisition of new sets of concurrent discriminations or in their retention of these sets, despite their equivalent difficulty with the preoperatively learned discrimination, which was impaired. These new sets were learned, and their retention tested, after the retention of preoperatively learned sets was assessed. While it is tempting to interpret these data as a retrograde effect in the absence of any anterograde effect, it is clear that retention of preoperatively learned sets was always tested first. Thus, it is possible that the deficit observed was merely a transient effect that resolved with time, or the animals may have learned to overcome a deficit behaviorally. It is possible that the impairment could be reinstated by more demanding testing procedures - for example, testing retention of larger or more confusable concurrent discrimination sets or testing over longer retention periods. These possibilities could be differentiated by testing retention of preoperatively learned sets after acquisition of a postoperatively learned set, as the current postoperative retention data show that the animals are capable of retaining two five-pair discriminations, even postoperatively. However, this finding of a deficit in postoperative retention of preoperatively learned discriminations is similar to that al- 
ready reported in the monkey (Buckley \& D. Gaffan, 1997; D. Gaffan \& Murray, 1992) although these authors also tested retention of preoperatively learned sets before postoperative acquisition and therefore cannot disambiguate our data further.

However, if replicated using a balanced order of testing, a retrograde effect in the absence of an anterograde effect could have many implications for the understanding of the role of perirhinal cortex in learning and memory. Although it has been suggested that this cortex is involved in visual recognition memory, the present results and a growing body of evidence from the monkey discussed above cast doubt on the simple version of this idea. However, it has been suggested that the perirhinal cortex may play a role in the identification of stimuli by being involved in the perceptual learning process by which stimuli may be uniquely identified (Eacott et al., 1994). However, evidence suggests that although perirhinal cortex may be important in this process, it is not critical, in that following ablation of the perirhinal cortex the process is less reliable but may still occur (Eacott et al., 1994). The present data may be understood in this way. During preoperative learning, the stimuli became recognizable by a process of perceptual learning which may involve the tuning of the response properties of cells. Such tuning of the response properties of neurons on the basis of experience has been found in and surrounding the perirhinal cortex in the monkey (Sakai \& Miyashita, 1991, 1994; Tanaka, 1993). Ablation of the perirhinal cortex may destroy this tuning and, as a result, the perirhinal group may have had to relearn aspects of this discrimination. However, postoperatively learned discriminations are never based on this perirhinal system and rely instead on an alternative, but in this case equally efficient, system which must reside elsewhere in the perceptual and memory system. Thus, no deficit is seen. This interpretation suggests that the deficit itself is not transient and that a deficit should be observed even when retention of preoperatively learned sets is delayed until after the learning of postoperative discrimination sets. Moreover, the deficit will be reinstatable, even with postoperatively learned discrimination sets, if the discrimination stresses the alternative system (Buckley \& D. Gaffan, 1997; Eacott et al., 1994). Subsequent experiments are in progress to resolve this question.

In summary, the present experiment revealed a deficit in retention of preoperatively learned concurrent discrimination sets following ablation of the perirhinal cortex in the rat. This result is similar to that reported in the monkey, but is inconsistently found in the rat.

\section{REFERENCES}

Astur, R. S., Mumby, D. G., \& Sutherland, R. J. (1995). Perirhinal cortex damage: Effects of acquisition and retention of object and place discriminations in rats. Society for Neuroscience Abstracts, 21, 1935.

BUCKLEY, M. J., \& GAFFAN, D. (1997). Impairment of visual object discrimination learning after perirhinal cortex ablation. Behavioral Neuroscience, 111, 467-475.

Bunsey, M., \& Eichenbaum, H. (1993). Critical role of the parahip- pocampal region for paired associates learning in rats. Behavioral Neuroscience, 107, 740-747.

Burwell, R. D., Witter, M. P., \& Amarel, D. G. (1995). Perirhinal and postrhinal cortices of the rat: A review of the neuroanatomical literature and comparison with findings from the monkey brain. Hippocampus, 5, 390-408.

Eacott, M. J., Gaffan, D., \& Murray, E. A. (1994). Preserved recognition memory for small sets and impaired stimulus identification for large sets, following rhinal cortex ablation in monkeys. European Journal of Neuroscience, 6, 466-478.

GAFFAN, D. (1994). Dissociated effects of perirhinal cortex ablation, fornix transection and amygdalectomy: Evidence for multiple memory systems in the primate temporal lobe. Experimental Brain Research, 99, 411-422.

GafFan, D., \& Murray, E. A. (1992). Monkeys with rhinal cortex lesions succeed in object discrimination learning despite 24-hour intertrial intervals and fail at match to sample despite double sample presentations. Behavioral Neuroscience, 106, 30-38.

Gaffan, E. A., \& EaCoTt, M. J. (1995). A computer-controlled maze environment for testing visual memory in the rat. Journal of Neuroscience Methods, 60, 23-37.

Horel, J. A., Pytko-Joiner, D. E., Voytko, M. L., \& Salsbury, K. (1987). The performance of visual tasks while segments of the inferotemporal cortex are suppressed by cold. Behavioural Brain Research, $23,29-42$.

Horel, J. A., VoytKo, M. L., \& Salsbury, K. G. (1984). Visual learning suppressed by cooling the temporal pole. Behavioral Neuroscience, 98, 310-324.

Kornecook, T. J., Lui, M., Duva, C. A., Anzarat, A., \& Pinel, J. P. J. (1995). Effects of perirhinal cortex lesions on object memory task in the rat. Society for Neuroscience Abstracts, 21, 1935.

Meunier, M., Bachevalier, J., Mishkin, M., \& Murray, E. A. (1993). Effects on visual recognition of combined and separate ablations of the entorhinal and perirhinal cortex in rhesus monkeys. Journal of Neuroscience, 13, 5418-5432.

Mumby, D. G., \& Pinel, J. P. J. (1994). Rhinal cortex lesions and object recognition in rats. Behavioral Neuroscience, 108, 1-8.

Murray, E. A., GafFan, D., \& Mishrin, M. (1993). Neural substrates of visual stimulus-stimulus association in rhesus monkeys. Journal of Neuroscience, 13, 4549-4561.

Otto, T., \& Eichenbaum, H. (1992). Complementary roles of the orbital prefrontal cortex and the perirhinal-entorhinal cortices in an odor-guided delayed-nonmatching to sample task. Behavioral Neuroscience, 106, 762-775.

Paxinos, G., \& Watson, W. (1986). The rat brain in stereotaxic coordinates. London: Academic Press.

SAKaI, K., \& MiYashita, Y. (1991). Neuronal organisation for the longterm memory of paired associates. Nature, 354, 152-155.

SAKAI, K., \& MiYASHITA, Y. (1994). Visual imagery: An interaction between memory retrieval and focal attention. Trends in Neurosciences, 17, 287-289.

TANaKa, K. (1993). Neuronal mechanisms of object recognition. Science, 262, 685-688.

WiIG, K. A., Booth, S. J., LuI, P., CoOPer, L., \& Bilkey, D. K. (1995). The effects of separate and combined lesions of perirhinal cortex, fornix and septum on memory in the rat. Society for Neuroscience Abstracts, 21, 1935.

ZHU, X. O., \& Brown, M. W. (1995). Changes in neuronal activity related to the repetition and relative familiarity of visual stimuli in rhinal and adjacent cortex of the anaesthetised rat. Brain Research, 689, 101-1 10.

Zhu, X. O., Brown, M. W., \& Aggleton, J. P. (1995). Neuronal signalling of information important to visual recognition in rat rhinal and neighbouring cortices. European Journal of Neuroscience, 7, 753-765.

Zola-Morgan, S., SQuire, L., Clower, R. P., \& Rempel, N. (1993). Damage to the perirhinal cortex exacerbated memory impairment following lesions to the hippocampal formation. Journal of Neuroscience, 13, 251-265.

(Manuscript received May 28, 1997; revision accepted for publication September 29, 1997.) 